\title{
The Epworth Sleepiness Scale in the Assessment of Sleep Disturbance in Veterans with Tinnitus
}

\author{
Yuan F. Liu, ${ }^{1}$ Jinwei Hu, ${ }^{1}$ Matthew Streelman, ${ }^{2}$ and O'neil W. Guthrie ${ }^{1,2,3}$ \\ ${ }^{1}$ Department of Otolaryngology-Head and Neck Surgery, Loma Linda University Medical Center, 11234 Anderson Street, \\ Loma Linda, CA 92354, USA \\ ${ }^{2}$ Loma Linda University School of Medicine, 11175 Campus Street, Loma Linda, CA 92350, USA \\ ${ }^{3}$ Loma Linda Veterans Affairs Medical Center, 11201 Benton Street, Loma Linda, CA 92357, USA
}

Correspondence should be addressed to Yuan F. Liu; yfang109@gmail.com

Received 21 October 2014; Accepted 19 December 2014

Academic Editor: Leonard P. Rybak

Copyright (C) 2015 Yuan F. Liu et al. This is an open access article distributed under the Creative Commons Attribution License, which permits unrestricted use, distribution, and reproduction in any medium, provided the original work is properly cited.

\begin{abstract}
Purpose. Tinnitus and sleep disturbance are prevalent in veterans, and a better understanding of their relationship can help with tinnitus treatment. Materials and Methods. Retrospective chart review of 94 veterans seen in audiology clinic between 2010 and 2013 is presented. Results. The mean age was 62 years, and 93 of 94 veterans were males. The majority (96\%) had hearing loss. The positive predictive value of the ESS for sleep disorder was $97 \%$ and the negative predictive value was $100 \%$. Veterans with a Tinnitus Handicap Inventory (THI) score $\geq 38$ had significantly higher Epworth Sleepiness Scale (ESS) scores compared to those with THI score $<38(P=0.006)$. The former had a significantly higher incidence of PTSD, anxiety, and sleep disorder. A subgroup of patients had normal sleep despite rising THI scores. Bilateral tinnitus, vertigo, and anxiety were found to be predictors of sleep disturbance. Conclusions. The ESS can be used as a tool in the initial assessment of sleep disorders in veterans with tinnitus. Higher tinnitus handicap severity is significantly associated with greater sleep disturbance. Optimal management of tinnitus may require concomitant treatment of sleep disorder, PTSD, anxiety, and depression.
\end{abstract}

\section{Introduction}

Tinnitus, defined as the perception of sound without an external source, affects an estimated 35 to 50 million Americans or 5 to $15 \%$ of the general population [1-5]. Although the majority of those affected by tinnitus habituate to the condition and do not seek treatment, 10-20\% experience tinnitus as a severe handicap [6].

Those who live with tinnitus may be burdened by comorbid stressors such as sleep disorders, depression, anxiety disorder, and suicidal ideation [7-9]. These conditions negatively impact many aspects of daily life, causing impairments in work and memory and reducing quality of life $[7,8,10]$.

Sleep disturbance is one of the most common comorbidities associated with tinnitus [11-13]. The prevalence of sleep disorders in patients with tinnitus varies from 25 to $77 \%$, with almost $50 \%$ reporting insomnia $[6,14-16]$. Hébert and Carrier found that patients suffering from tinnitus reported greater sleep difficulties compared to controls, specifically in sleep efficiency and sleep quality [17]. Using questionnaires, other studies have found a higher prevalence of sleep complaints in tinnitus sufferers compared to the general population $[13,18]$. It has also been shown that sleep disturbance strongly predicts lower tinnitus tolerance, while successful treatment of tinnitus results in fewer sleep complaints [11, 17, 19-21].

There is no definitive explanation of how tinnitus may lead to sleep disturbance. It has been postulated that when environmental noise wanes at night, tinnitus awareness may rise with the initiation of unhelpful thoughts, mood changes, and physical reactions, thereby initiating a cycle of anxiety, arousal, and distress [22]. Whatever the mechanism, it is important to note that insomnia is associated with functional impairment and decreased quality of life, especially among subjects with tinnitus who are older than 50 years of age $[2,11,14,23]$.

There are currently more than 20 million US veterans, a predominantly male, aging population with a history of 
noise exposure that is served by the Veterans Health Administration [24]. This cohort is especially prone to developing tinnitus, which has an increasing prevalence with age, and affects men more commonly than women [4, 25-27].

With the aid of the Epworth Sleepiness Scale (ESS), Tinnitus Handicap Inventory (THI), Tinnitus and Hearing Survey (THS), and Tinnitus Problem Checklist (TPC), we sought to address the following questions: can the ESS be used in the initial assessment of sleep disturbance in veterans with tinnitus? If so, among veterans with different degrees of tinnitus handicap severity, is there a difference in sleep disturbance? Are there any differences in demographics, hearing profiles, or psychiatric comorbidities that can distinguish veterans with different degrees of tinnitus? Can any characteristics of veterans with tinnitus help predict sleep disturbance?

\section{Materials and Methods}

2.1. Data Gathering. A retrospective chart review was conducted at the Veterans Affairs Medical Center (VAMC) in Loma Linda, CA, on patients with tinnitus complaints. The Loma Linda VAMC serves about 67,000 veterans living in the San Bernardino and Riverside Counties. Audiologic, otologic, and psychiatric data were retrieved from the computerized patient record system (CPRS). Charts for 117 patients were reviewed and 94 patients who had at least a completed Tinnitus Handicap Inventory and pure-tone audiometry data were included in the final analyses. These charts were from patients who visited the Loma Linda VAMC audiology clinic between 2010 and 2013. The data collected from CPRS included ICD-9 codes along with qualitative (e.g., type, severity, and degree) and quantitative (e.g., parametric results from diagnostic and screening tests) data when available. All procedures were reviewed and approved by the Institutional Review Board at the Loma Linda VAMC.

2.2. Epworth Sleepiness Scale (ESS). The ESS is a standardized tool used to measure daytime sleepiness. It contains 8 questions, each scoring $0-3$ with increasing number signifying higher chance of "dozing" while engaged in specific activities of daily life. A score of less than 10 is generally considered clinically normal [28-30]. The ESS was developed in 1991, was modified in 1997, and has become the most frequently used method worldwide for assessing daytime sleepiness due to its reliability, consistency, and ease of use $[28,31,32]$.

2.3. Tinnitus Handicap Inventory (THI). The THI is a survey containing 25 questions. Each question is worth up to 4 points, with 4 for "yes," 2 for "sometimes," and 0 for "no," for a total of 100 points. A score of $0-16$ denotes no handicap from tinnitus, 18-36 denotes mild handicap, and 38-56 denotes moderate handicap, and higher scores denote severe handicap. The questions can be divided into three categories which contribute to three subscales: functional (11 questions, 48 points), emotional ( 9 questions, 32 points), and catastrophic (5 questions, 20 points). The functional score reflects the effect of tinnitus on mental, social, occupational, and physical functioning. The emotional score reflects affective response to tinnitus. The catastrophic score reflects desperation, inability to escape, perception of having a terrible disease, lack of control, and inability to cope with tinnitus. The THI has been validated for being a robust tool for measuring the effect of tinnitus on daily life, with a score of 38 or higher suggesting significant tinnitus requiring intervention [33-36].

2.4. Tinnitus and Hearing Survey (THS). The THS contains 10 questions; each scored 0-4 for increasing tinnitus, hearing, and/or sound intolerance severity. Four questions are for tinnitus, 4 questions are for hearing loss, and 1 question is for hyperacusis. A final, unscored question is answered yes or no regarding severity of hyperacusis. The THS is a nonvalidated instrument that is used to rapidly determine how much of a reported problem is due to tinnitus, hearing, and/or hyperacusis. This measurement is often necessary because tinnitus patients tend to confuse hearing problems with tinnitus problem. Therefore, the THS is an efficient screening tool that allows clinicians to differentiate which of the three problems (tinnitus, hearing, and/or hyperacusis) is most troublesome to the patient $[37,38]$. A total score of 3 or more on the tinnitus portion of the survey may suggest a need for clinical intervention [38-41].

2.5. Tinnitus Problem Checklist (TPC). The TPC is used to identify bothersome tinnitus situations [38]. The patient is instructed to select a situation where tinnitus is most bothersome. The patient then chooses the first, second, and third most bothersome tinnitus situations. The selections include falling asleep at night, staying asleep at night, waking up in the morning, reading, working on the computer, relaxing in my recliner, napping during the day, planning activities, driving, and others (where the patient can report a situation that is not listed among the choices).

2.6. Statistics. Analyses of continuous variables were performed using 2-tailed, unequal variance, Student's $t$-test, while analyses of nominal and ordinal variables were performed using Fisher's exact test. Fisher's exact test was chosen in place of Pearson's chi-squared test in order to eliminate limitations dealing with low counts of "yes" or "no" for some variables. Analysis of variance (ANOVA) was used to compare more than 2 groups of continuous variables. Linear regression was performed using Minitab 17. Odds ratios calculated from coefficients of the linear regression model are reported with 95\% confidence intervals (CI). Other analyses were performed using Microsoft Excel 2010. Means are reported with \pm standard deviations (SD). For all variables, a $P$ value $<0.05$ was considered to be statistically significant.

\section{Results}

Demographic, otologic, audiologic, and psychiatric profiles are presented in Table 1. Of 94 veterans, 93 (99\% patients) were male and 1 was female. The mean age was 62 years. Veterans from the Army, Navy, and Air Force were represented. Military noise was the predominant source of noise 
TABLE 1: Demographic, otologic, audiologic, and psychiatric profiles. SD: standard deviation, normal ear: tympanic membrane and external auditory canal, 3F-PTA: 3-frequency-pure-tone average (500, 1,000, and 2,000 Hz), SRT: speech reception threshold, WDS: word discrimination score, and PTSD: posttraumatic stress disorder.

\begin{tabular}{|c|c|c|c|}
\hline Total of 94 patients & Patients & $\%($ mean $\pm S D$, median, $\min -\max )$ & Unknown \\
\hline \multicolumn{4}{|l|}{ Sex } \\
\hline Male & 93 & 99 & 0 \\
\hline Female & 1 & 1 & 0 \\
\hline Age & & $(62 \pm 10.3,64,31-84)$ & \\
\hline \multicolumn{4}{|l|}{ Service } \\
\hline Army & 35 & & \\
\hline Navy & 13 & & 41 \\
\hline Air force & 5 & & \\
\hline \multicolumn{4}{|l|}{ Noise exposure } \\
\hline Military & 67 & & 24 \\
\hline Occupational & 36 & & 25 \\
\hline Recreational & 21 & & 39 \\
\hline All & 69 & & 21 \\
\hline Previous hearing aids use & 18 & & 7 \\
\hline Vertigo & 23 & & 20 \\
\hline Middle ear symptoms & 14 & & 9 \\
\hline Ear surgery & 7 & & 2 \\
\hline Ear injury & 9 & & 23 \\
\hline Ear infection & 10 & & 45 \\
\hline Ear pain & 16 & & 36 \\
\hline Aural fullness or pressure & 18 & & 32 \\
\hline \multicolumn{4}{|l|}{ Normal ear } \\
\hline Left & 91 & 97 & 0 \\
\hline Right & 90 & 96 & 0 \\
\hline \multicolumn{4}{|l|}{ Left hearing loss } \\
\hline Sensorineural & 87 & 93 & \multirow{2}{*}{2} \\
\hline Mixed & 2 & 2 & \\
\hline \multicolumn{4}{|l|}{ Right hearing loss } \\
\hline Sensorineural & 86 & 91 & \multirow{2}{*}{2} \\
\hline Mixed & 2 & 2 & \\
\hline \multicolumn{4}{|l|}{ 3F-PTA } \\
\hline Left & 94 & $(28 \pm 14.3,25,6.7-83.3)$ & 0 \\
\hline Right & 94 & $(27 \pm 15.3,25,5-93)$ & 0 \\
\hline \multicolumn{4}{|l|}{ SRT } \\
\hline Left & 92 & $(25 \pm 13.5,25,0-80)$ & 2 \\
\hline Right & 91 & $(24 \pm 15.1,20,0-100)$ & 3 \\
\hline \multicolumn{4}{|l|}{ WDS } \\
\hline Left & 92 & $(91 \pm 12.7,95,0-100)$ & 2 \\
\hline Right & 92 & $(90 \pm 15.1,96,20-100)$ & 2 \\
\hline Sleep disorder & 37 & 39 & 3 \\
\hline PTSD & 77 & 82 & 4 \\
\hline Anxiety & 75 & 80 & 2 \\
\hline Depression & 57 & 61 & 1 \\
\hline Claustrophobia & 9 & & 48 \\
\hline
\end{tabular}

exposure, affecting 67 (71\%) patients. Vertigo was the most common otologic symptom, affecting $23(24 \%)$ patients. The majority of veterans $(90,96 \%)$ had some degree of hearing loss in one ear or the other, and most of them $(88,94 \%)$ were diagnosed with sensorineural hearing loss. Sleep disorder, based on ICD-9 diagnoses by Behavioral Health Medicine, was found in 37 (39\%) veterans. Furthermore, posttraumatic stress disorder (PTSD) was found in 77 (82\%) patients, 
TABLE 2: Tinnitus characteristics.

\begin{tabular}{|c|c|c|c|}
\hline Total of 94 patients & Patients & $\%($ mean $\pm \mathrm{SD}$, median, $\min -\max )$ & Unknown \\
\hline \multicolumn{4}{|l|}{ Tinnitus quality } \\
\hline Bilateral & 74 & & \multirow{2}{*}{13} \\
\hline Unilateral & 7 & & \\
\hline Constant & 56 & & \multirow{2}{*}{14} \\
\hline Intermittent & 24 & & \\
\hline \multicolumn{4}{|l|}{ Tinnitus duration } \\
\hline$\leq 1$ year & 3 & & \multirow{7}{*}{37} \\
\hline $2-10$ years & 20 & & \\
\hline $11-20$ years & 4 & & \\
\hline $21-30$ years & 17 & & \\
\hline $31-40$ years & 5 & & \\
\hline $41-50$ years & 7 & & \\
\hline$>50$ years & 1 & & \\
\hline \multicolumn{4}{|l|}{ Tinnitus Handicap Inventory } \\
\hline Total score & 94 & $(57 \pm 23.5,59,4-96)$ & 0 \\
\hline Functional score & 94 & $(29 \pm 11.4,31,0-44)$ & 0 \\
\hline Emotional score & 94 & $(17 \pm 9.1,18,0-32)$ & 0 \\
\hline Catastrophic score & 94 & $(11 \pm 4.8,10,2-20)$ & 0 \\
\hline \multicolumn{4}{|l|}{ Tinnitus and Hearing Survey } \\
\hline Tinnitus score & 94 & $(10 \pm 4.0,10.5,0-16)$ & 0 \\
\hline Hearing score & 94 & $(11 \pm 4.5,11.5,0-16)$ & 0 \\
\hline Hyperacusis score & 94 & $(1.9 \pm 1.6,2,0-4)$ & 0 \\
\hline \multicolumn{4}{|c|}{ Tinnitus Problem Checklist: most bothersome situation } \\
\hline Falling asleep at night & 44 & & \multirow{4}{*}{20} \\
\hline Staying sleep & 5 & & \\
\hline Waking up in the morning & 2 & & \\
\hline Napping during the day & 0 & & \\
\hline Epworth Sleepiness Scale score & 91 & $(10 \pm 5.4,9,1-22)$ & 3 \\
\hline
\end{tabular}

anxiety in $75(80 \%)$ patients, and depression in 57 (61\%) patients.

3.1. Tinnitus Characteristics. Features specific to tinnitus are presented in Table 2. Most veterans (74, 79\%) had bilateral rather than monaural tinnitus, which was predominantly constant (versus intermittent) in $56(60 \%)$ patients. More patients $(20,21 \%)$ began suffering from tinnitus within the last 1 to 10 years compared to any other time point. The most bothersome tinnitus situation was falling asleep at night (44 patients, $47 \%$ ), followed by staying asleep at night (5 patients, $5 \%$ ) and waking up in the morning (2 patients, $2 \%$ ).

The average total THI score was $57 \pm 23.5$ (median 59, range 4-96), suggesting that this population of veterans fell within the severe level (score $>56$ ) of tinnitus handicap and should undergo clinical intervention. The mean score on the tinnitus portion of the THS was $10 \pm 4.0$ (median 10.5 , range $0-16$ ), also indicating that this population had clinically significant tinnitus. The mean THS hearing score was $11 \pm 4.5$ (median 11.5 , range $0-16$ ), signifying the presence of concurrent hearing loss. The mean THS hyperacusis score was $1.9 \pm 1.6$ (median 2 , range $0-4$ ), implying a moderate problem with hyperacusis as well. The mean ESS score was $10 \pm 5.4$ (median 9, range 1-22), lying at the border of normal and abnormal daytime sleepiness. Thirty-eight (42\%) patients had an ESS score of 10 or more.

3.2. The Epworth Sleepiness Scale as a Measure of Sleep Disturbance. Of those veterans with sleep disorder, the average ESS score was $15.4 \pm 3.6$, compared to $6.0 \pm 2.2$ for those without sleep disorder $(P<0.001)$. Using an ESS score of 10 as a cutoff for clinical significance, all patients were divided into 2 groups: ESS score $<10$ and ESS score $\geq 10$. Sleep disorder was found in 0 of 53 patients in the former group and 37 of $38(97 \%)$ patients in the latter $(P<0.001)$. The positive predictive value (PPV) of the ESS for sleep disorder given the above cutoff was $97 \%$, and the negative predictive value (NPV) was $100 \%$. Thus, we elected to use the ESS score as a measure of sleep disturbance in the subsequent analyses.

\subsection{Differences in Sleep Disturbance and Other Characteristics} among Tinnitus Severity Groups. Based on the THI total score, veterans were divided into 4 tinnitus handicap severity groups: no tinnitus handicap (0-16), mild (18-36), moderate $(38-56)$, and severe $(>56)$. The no tinnitus handicap group had a mean ESS of $5.8 \pm 2.9$, the mild group $7.8 \pm 1.6$, the moderate group $10.1 \pm 4.3$, and the severe group 10.7 \pm 6.3. ANOVA testing was performed to assess whether 
TABLE 3: Significant differences between tinnitus severity groups. THI: Tinnitus Handicap Inventory, SD: standard deviation, PTSD: posttraumatic stress disorder, R WDS: right ear word discrimination score, and THS: Tinnitus and Hearing Survey.

\begin{tabular}{|c|c|c|c|c|c|}
\hline \multirow{2}{*}{ Factor } & \multicolumn{2}{|c|}{ THI $<38$} & \multicolumn{2}{|c|}{$\mathrm{THI} \geq 38$} & \multirow{2}{*}{$P$ value } \\
\hline & Incidence & Mean \pm SD & Incidence & Mean \pm SD & \\
\hline PTSD & $58.80 \%$ & & $91.80 \%$ & & 0.002 \\
\hline Anxiety & $41.20 \%$ & & $90.70 \%$ & & $<0.001$ \\
\hline Sleep disorder & $11.80 \%$ & & $47.30 \%$ & & 0.007 \\
\hline R WDS & & $96.0 \pm 4.2$ & & $89.2 \pm 16.5$ & 0.002 \\
\hline \multicolumn{6}{|l|}{ THS score } \\
\hline Tinnitus & & $4.5 \pm 3.1$ & & $11.3 \pm 3.0$ & $<0.001$ \\
\hline Hearing & & $6.5 \pm 5.2$ & & $11.7 \pm 3.7$ & 0.001 \\
\hline Hyperacusis & & $0.9 \pm 1.0$ & & $2.2 \pm 1.6$ & $<0.001$ \\
\hline
\end{tabular}

TABLE 4: Correlation coefficients for Tinnitus Handicap Inventory (THI) total score and Epworth Sleepiness Scale score.

\begin{tabular}{lc}
\hline Group & $R^{2}$ \\
\hline All patients & 0.046 \\
$\mathrm{THI} \leq 46$ & 0.332 \\
$\mathrm{THI}>46$ and $\mathrm{ESS} \geq 10$ & 0.271 \\
$\mathrm{THI}>46$ and $\mathrm{ESS}<10$ & 0.213 \\
\hline
\end{tabular}

there was a difference in mean ESS score among the groups and no significant difference was found $(P=0.071)$. We proceeded with the analysis by aggregating the patients into fewer tinnitus handicap severity groups.

Since a THI total score of 38 was suggested as the cutoff for clinical significance, we divided the patients into 2 groups: THI total score $<38$ and $\geq 38$. The former group had a mean ESS score of 6.8 and the latter a mean of 9.6 $(P=0.006)$. This is shown in Figure 1. Subsequently, we searched for a difference between the 2 tinnitus groups with respect to each variable listed in Tables 1 and 2. The group with THI total score $<38$ was found to have a significantly lower incidence of PTSD, anxiety, and sleep disorder, higher average right ear word discrimination score, and lower THS tinnitus, hearing, and hyperacusis scores. Notably, there was no difference in other measures of hearing loss, and there was a near-significant difference in rate of depression (39\% in THI $<38$ group, $67 \%$ in THI $\geq 38$ group, $P=0.057$ ). Table 3 summarizes these significant factors.

A scatterplot was constructed to evaluate the global association between tinnitus (total THI score) and sleep disturbance (ESS score), as shown in Figure 2. On visual inspection, a portion of the data appeared to drift upwards while a portion appeared to plateau or drift slightly downwards at around an ESS score of 10. Taking this observation into account, the data was partitioned into 3 groups to more clearly define correlational attributes between these variables: patients with THI scores $\leq 46$, designated as "normal"; patients with THI scores $>46$ and ESS scores $\geq 10$, designated as "high-high"; and patients with THI scores $>46$ and ESS scores $<10$, designated as "high-normal." Table 4 provides a summary of correlation coefficients $\left(R^{2}\right)$. Comparing the

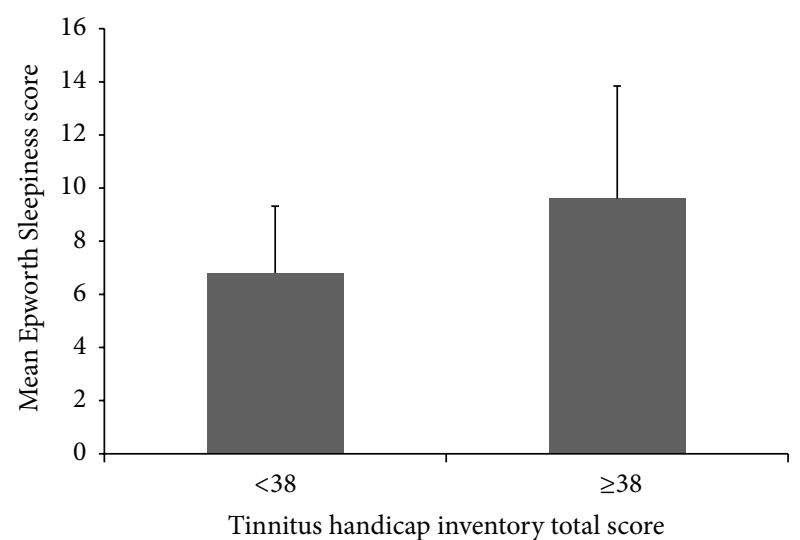

FIgure 1: Mean Epworth Sleepiness Scale (ESS) score for Tinnitus Handicap Inventory (THI) total score $<38$ and $\geq 38$. Vertical bars with caps indicate standard deviations.

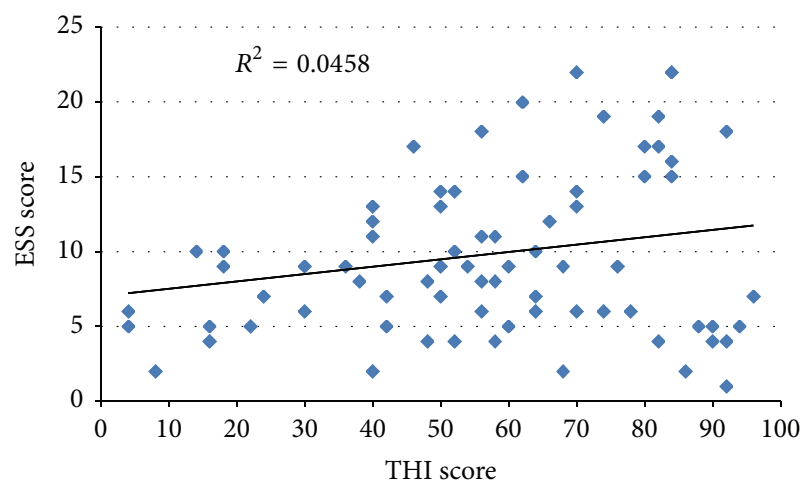

FIGURE 2: Scatterplot of Tinnitus Handicap Inventory (THI) score versus Epworth Sleepiness Scale (ESS) score.

high-high group and the high-normal group in terms of characteristics listed in Tables 1 and 2, the former had a higher percentage of bilateral tinnitus (100\% versus $80 \%, P=0.025)$, lower left ear word discrimination score (89.8 versus 93.8 , $P=0.049)$, and higher THS hearing score (13.1 versus 11.4, $P=0.028)$. 
3.4. Predictors of Sleep Disturbance among Tinnitus Patients. To reveal which demographic, otologic, audiologic, or psychiatric variables are associated with sleep disturbance in tinnitus patients, we performed linear regression using all factors listed in Tables 1 and 2 (except sleep disorder) as independent variables to predict sleep disturbance in terms of ESS score $<10$ or $\geq 10$. Three variables were found to be significantly associated with an ESS score of $\geq 10$ : bilateral tinnitus $(P=0.006)$, vertigo $(P=0.036)$, and anxiety $(P=$ $0.007)$. The odds ratios were 1.80 (95\% CI 1.20, 2.70), 1.30 (95\% CI 1.02, 1.66), and 1.62 (95\% CI 1.15, 2.27), respectively. The adjusted correlation coefficient $\left(R^{2}\right)$ was 0.223 .

\section{Discussion}

Noise, or a perception thereof, can be a natural obstacle to resting and initiating sleep. Many studies have examined the relationship between tinnitus and sleep. An Italian group found that $54 \%$ of study patients had sleep disorders and that maintaining sleep was the predominant complaint [22]. Lasisi and Gureje studied 1302 elderly subjects and found that $11.4 \%$ had tinnitus, with $51.9 \%$ of those with tinnitus reporting insomnia, compared to only $33.8 \%$ of those without tinnitus $(P=0.002)[16]$. In the same study, it was found that difficulty falling asleep and morning wakefulness were significantly associated with tinnitus. Likewise, a large cross-sectional survey of 4,705 tinnitus patients conducted in Germany discovered that nearly $77 \%$ had sleep difficulty and $46.4 \%$ attributed the cause of their sleep disturbance to tinnitus [6].

Clinical studies have linked tinnitus specifically to decreased sleep quality $[6,15,19,20]$. Alster et al. used the Mini Sleep Questionnaire to compare 80 tinnitus patients and a control group (both groups representing military personnel without major psychiatric comorbidities), to find that $77 \%$ of the tinnitus group had worse scores, particularly in prolonged sleep latency, microarousals, and morning fatigue [19]. Attanasio et al. reported that sleep quality decreased with an increase in self-reported tinnitus severity and that there was a significant alteration in all stages of sleep in tinnitus patients, with greater periods of stage 1 and 2 sleep than stages 3, 4, and REM sleep [42]. Similarly, other studies found lower spectral power in the delta frequency band that appears in sleep stages 3 and 4 of tinnitus patients, which correlated with subjective sleep complaints $[43,44]$.

Despite the lack of a proven mechanism by which tinnitus directly causes sleep disturbance, some theories have been formulated. Tinnitus may interfere with sleep by the amplification of internally perceived noise during times of low environmental noise. Also, increased awareness of tinnitus before falling asleep may lead to increased focus on tinnitus and undue anxiety which may lengthen sleep onset latency $[6,22]$. On a physiological level, tinnitus has been suggested to lead to sleep disturbance through cortical pathways involving the auditory cortex as well as other nuclei. In the gerbil animal model, tinnitus was induced by salicylate injection or loud noise exposure, and c-fos, a marker of neuronal activity, was screened for and found to be consistently expressed in the auditory cortex, the frontal cortex, areas responsible for behavioral and physiological stress reactions, and areas controlling autonomic function [45]. A similar pattern of c-fos expression was found in the brains of rats that were exposed to a stressor which led to increased sleep latency, decreased nonrapid eye movement (NREM) and REM sleep, and increased sleep fragmentation [46]. These and other animal studies have led to the notion that auditory information enters the amygdala from either the auditory cortex or the thalamic auditory relay nucleus and is thought to be enhanced in people with tinnitus. In doing so, areas of the amygdala are activated depending on the emotional significance of the stimuli, thereby eliciting a defensive response associated with fear [6].

Patients treated at Veterans Affairs (VA) Hospitals are particularly susceptible to tinnitus and its consequences. Veterans are exposed to high level of occupational noise, with increasing duration of military service correlating with lower hearing sensitivity [47]. Increased noise exposure is the most common cause of tinnitus [48]. As such, it was estimated in 2004 that 3 to 4 million American veterans were suffering from tinnitus and that tinnitus is regarded as one of the most disabling conditions caused by military service [49]. Its predominantly male sex and increased age make the VA population more vulnerable to tinnitus [24]. When the VA population is divided into 5-year age intervals, the greatest number of veterans lies between the ages of 65 and 69 [24]. Subjective tinnitus annoyance seems to increase in men with age, though this may partially be attributed to increased hearing problems with age [50]. For those older than 50 years, the likelihood of problems with sleep maintenance doubles [6].

Thirty-nine percent of our veteran population had an ICD-9 diagnosis of sleep disorder. This is within the range reported in the literature, as discussed previously (about 25$77 \%)$. An ESS score of 10 or above has been used as a cutoff for clinically abnormal daytime sleepiness. We found that, with this cutoff, the ESS was able to predict the presence of sleep disorder with a positive predictive value of $97 \%$ and negative predictive value of $100 \%$. Therefore, the ESS can be an accurate tool for assessing the presence of sleep disturbance in veterans with tinnitus.

When veterans were divided into 4 tinnitus handicap severity groups, from no tinnitus handicap to severe, we did not find a significant difference in mean ESS score among the groups. However, the no tinnitus handicap and mild tinnitus groups had "normal" range $(<10)$ (mean ESS scores 5.8 and 7.8 , resp.), while the moderate and severe groups showed "abnormal" range ( $\geq 10)$ (ESS scores 10.1 and 10.7, resp.). Given the positive trend, there were possibly an insufficient number of subjects to detect a small difference in ESS scores. However, we were able to find a significant difference in ESS scores when patients were divided into 2 larger tinnitus handicap severity groups: THI $<38$ and THI $\geq 38$. We explored significant differences between these 2 groups and found that the THI $<38$ group had a lower incidence of PTSD (59\% versus 92\%), anxiety (41\% versus 91\%), and sleep disorder (12\% versus $47 \%)$. Fagelson found that the incidence of PTSD was $34 \%$ in a veteran population suffering from tinnitus, compared to our finding of $82 \%$ overall [51]. In primary care clinics, $5-13 \%$ of veterans have been found to 
have PTSD [52]. The general veteran population has been reported to have a lifetime PTSD prevalence of $31 \%$ in males and $27 \%$ in females [53]. Not only did our veteran population have a much higher rate of PTSD, but we also encountered a dramatic increase in PTSD with an increase in tinnitus handicap severity. This suggests that there is a positive linear or perhaps even exponential relationship between PTSD and tinnitus. Furthermore, there may be a synergistic effect involving tinnitus, PTSD, and sleep disorder in which the disease entities worsen one another's impact. Anxiety, which is closely related to PTSD, likely has a similar influence. Of note was the lower incidence of depression in the THI $<38$ group compared to the $\mathrm{THI} \geq 38$ group (39\% versus $67 \%$ ). We suspect that depression also influences tinnitus, but perhaps our population was not large enough to capture the effect.

Interestingly, we discovered three distinct groups of patients as a function of THI and ESS scores. The group with THI total score $\leq 46$ shared approximately $33 \%$ of the variance between THI and ESS scores. Above a score of 46 , there was a group with increasing tinnitus handicap as well as increasing daytime sleepiness and a group with increasing tinnitus handicap and steady or decreasing daytime sleepiness. Coincidentally, the ESS cutoff score between the 2 groups approximately corresponds to the borderline of where sleepiness becomes clinically significant [28]. Previous documentation of this phenomenon was not found in the literature. The high-high group (THI > 46 and ESS $\geq 10$ ) had a significantly higher percentage of bilateral tinnitus, lower left ear word discrimination score, and higher THS hearing score. No specific evidence relating tinnitus laterality to sleep disturbance was found in the literature. One can only speculate that unilateral tinnitus can be partially offset by head positioning on the pillow during sleep initiation. The low left ear word discrimination score combined with high THS hearing score might suggest retrocochlear involvement, but, in the absence of specific site-of-lesion assessments, such notions remain speculative.

Finally, we wanted to determine which factors may be used to predict sleep disturbance in a veteran population suffering from tinnitus. We found that bilateral tinnitus, vertigo, and anxiety were associated with ESS score $\geq 10$. There is some evidence that lack of sleep may trigger migraines and migrainous vertigo, but no literature was found on a direct relationship between vertigo and sleep disturbance in tinnitus patients [54]. Anxiety has been well documented as a contributor to sleep disturbance $[55,56]$. It has also been reported to correlate with tinnitus handicap severity [57].

It appears that PTSD, anxiety, and depression all exacerbate, to some degree, sleep disturbance and tinnitus in the veteran population. There are likely other psychiatric comorbidities that also play a role but were not studied in this paper. This underscores the importance of treatment for these disorders for patients with tinnitus. Antidepressants, such as selective serotonin reuptake inhibitors (SSRIs), have been shown to be effective in psychiatric disorders such as PTSD and depression [58, 59]. There is also evidence for sleep improvement when they are effectively used [60]. However, a 2006 Cochrane Review showed insufficient evidence that antidepressants improve tinnitus [61]. Perhaps future prospective studies can focus on the efficacy of antidepressants in veterans who are prone to, and present with, both psychiatric disorders and tinnitus, along with sleep disorder. Another avenue for research would be to compare the efficacy of psychotherapy versus antidepressants. What is the cause and effect relationship among tinnitus, sleep, and psychiatric comorbidities? The fact that many patients present with these disorders simultaneously, the lack of objective measurements for severity, and overlapping disease mechanisms all contribute to the complexity of this question. Future physiological studies using more objective tools such as polysomnography, brain MRI, and other imaging modalities, and molecular markers may better elucidate the relationship.

\section{Conclusions}

The ESS can be used as a tool in the initial assessment of sleep disorders in veterans with tinnitus, with a PPV of $97 \%$ and NPV of $100 \%$. There is a positive correlation between tinnitus and sleep disturbance in veterans, as has been shown in the general populations. However, we discovered a higher rate of PTSD (82\%) in veterans with tinnitus than previously reported in the literature and found that veterans may suffer from comorbidities such as PTSD, anxiety, and depression which appear to act synergistically to worsen sleep disturbance and/or tinnitus. There appears to be a distinct population of veterans who have high tinnitus handicap severity, yet no sleep disturbance. Unlike this group, those with high tinnitus handicap severity and abnormal sleep perceive increased hearing loss despite a lack of audiometric evidence, the reason for which is unclear. Furthermore, vertigo and bilateral tinnitus seem to contribute to sleep disturbance in tinnitus patients, the mechanism of which has not been explored. Our study supports a need for multifaceted management of tinnitus in veterans, including treatment for psychiatric comorbidities and sleep disturbance, in order to achieve an improvement in tinnitus handicap severity.

\section{Conflict of Interests}

The authors declare that there is no conflict of interests regarding the publication of this paper.

\section{Disclosure}

The first author had full access to all of the data in the study and takes responsibility for the integrity of the data and the accuracy of the data analysis.

\section{Acknowledgment}

The authors would like to acknowledge and thank Dr. Najeeb A. Shirwany for his critical evaluation of this paper.

\section{References}

[1] R. R. Coles, "Epidemiology of tinnitus: (1) prevalence," The Journal of Lryngology and Otology, vol. 98, supplement S9, pp. 7-15, 1984 . 
[2] A. Axelsson and A. Ringdahl, "Tinnitus-a study of its prevalence and characteristics," British Journal of Audiology, vol. 23, no. 1, pp. 53-62, 1989.

[3] J. A. Henry, K. C. Dennis, and M. A. Schechter, "General review of tinnitus: prevalence, mechanisms, effects, and management," Journal of Speech, Language, and Hearing Research, vol. 48, no. 5, pp. 1204-1235, 2005.

[4] S. J. Veterans, Tinnitus: Theory and Management, BC Decker, Lewiston, NY, USA, 2008.

[5] J. Shargorodsky, G. C. Curhan, and W. R. Farwell, "Prevalence and characteristics of tinnitus among US adults," The American Journal of Medicine, vol. 123, no. 8, pp. 711-718, 2010.

[6] E. Wallhäusser-Franke, M. Schredl, and W. Delb, “Tinnitus and insomnia: is hyperarousal the common denominator?" Sleep Medicine Reviews, vol. 17, no. 1, pp. 65-74, 2013.

[7] R. F. F. Cima, J. W. S. Vlaeyen, I. H. L. Maes, M. A. Joore, and L. J. C. Anteunis, "Tinnitus interferes with daily life activities: a psychometric examination of the tinnitus disability index," Ear and Hearing, vol. 32, no. 5, pp. 623-633, 2011.

[8] B. Langguth, "A review of tinnitus symptoms beyond 'ringing in the ears': a call to action," Current Medical Research \& Opinion, vol. 27, no. 8, pp. 1635-1643, 2011.

[9] J. M. Malouff, N. S. Schutte, and L. A. Zucker, “Tinnitus-related distress: a review of recent findings," Current Psychiatry Reports, vol. 13, no. 1, pp. 31-36, 2011.

[10] S. Rossiter, C. Stevens, and G. Walker, "Tinnitus and its effect on working memory and attention," Journal of Speech, Language, and Hearing Research, vol. 49, no. 1, pp. 150-160, 2006.

[11] R. S. Tyler and L. J. Baker, "Difficulties experienced by tinnitus sufferers," The Journal of Speech and Hearing Disorders, vol. 48, no. 2, pp. 150-154, 1983.

[12] L. Sanchez and D. Stephens, "A tinnitus problem questionnaire in a clinic population," Ear and Hearing, vol. 18, no. 3, pp. 210217, 1997.

[13] R. Asplund, "Sleepiness and sleep in elderly persons with tinnitus," Archives of Gerontology and Geriatrics, vol. 37, no. 2, pp. 139-145, 2003.

[14] R. L. Folmer and S. E. Griest, "Tinnitus and insomnia," American Journal of Otolaryngology, vol. 21, no. 5, pp. 287-293, 2000.

[15] E. Marciano, L. Carrabba, P. Giannini et al., "Psychiatric comorbidity in a population of outpatients affected by tinnitus," International Journal of Audiology, vol. 42, no. 1, pp. 4-9, 2003.

[16] A. O. Lasisi and O. Gureje, "Prevalence of insomnia and impact on quality of life among community elderly subjects with tinnitus," Annals of Otology, Rhinology \& Laryngology, vol. 120, no. 4, pp. 226-230, 2011.

[17] S. Hébert and J. Carrier, "Sleep complaints in elderly tinnitus patients: a controlled study," Ear and Hearing, vol. 28, no. 5, pp. 649-655, 2007.

[18] R. S. Hallam, "Correlates of sleep disturbance in chronic distressing tinnitus," Scandinavian Audiology, vol. 25, no. 4, pp. 263-266, 1996.

[19] J. Alster, Z. Shemesh, M. Ornan, and J. Attias, "Sleep disturbance associated with chronic tinnitus," Biological Psychiatry, vol. 34, no. 1-2, pp. 84-90, 1993.

[20] K. Eysel-Gosepath and O. Selivanova, "Characterization of sleep disturbance in patients with tinnitus," Laryngo- Rhino- Otologie, vol. 84, no. 5, pp. 323-327, 2005.

[21] T. Crönlein, B. Langguth, P. Geisler, and G. Hajak, "Tinnitus and insomnia," Progress in Brain Research, vol. 166, pp. 227-233, 2007.
[22] A. B. Fioretti, M. Fusetti, and A. Eibenstein, "Association between sleep disorders, hyperacusis and tinnitus: evaluation with tinnitus questionnaires," Noise and Health, vol. 15, no. 63, pp. 91-95, 2013.

[23] S. C. Jakes, R. S. Hallam, C. Chambers, and R. Hinchcliffe, "A factor analytical study of tinnitus complaint behaviour," Audiology, vol. 24, no. 3, pp. 195-206, 1985.

[24] US Department of Veterans Affairs, "Veteran Population," Veteran Population Statistics, 2013, http://www.va.gov/vetdata/ Veteran_Population.asp.

[25] C. S. Hankin, A. Spiro III, D. R. Miller, and L. Kazis, "Mental disorders and mental health treatment among U.S. Department of Veterans Affairs outpatients: the Veterans Health Study," The American Journal of Psychiatry, vol. 156, no. 12, pp. 1924-1930, 1999.

[26] N. Ahmad and M. Seidman, "Tinnitus in the older adult: epidemiology, pathophysiology and treatment options," Drugs \& Aging, vol. 21, no. 5, pp. 297-305, 2004.

[27] D. M. Nondahl, K. J. Cruickshanks, G.-H. Huang et al., "Tinnitus and its risk factors in the Beaver Dam offspring study," International Journal of Audiology, vol. 50, no. 5, pp. 313-320, 2011.

[28] M. W. Johns, "A new method for measuring daytime sleepiness: the Epworth sleepiness scale," Sleep, vol. 14, no. 6, pp. 540-545, 1991.

[29] M. W. Johns, "Reliability and factor analysis of the Epworth Sleepiness Scale," Sleep, vol. 15, no. 4, pp. 376-381, 1992.

[30] T. B. Kendzerska, P. M. Smith, R. Brignardello-Petersen, R. S. Leung, and G. A. Tomlinson, "Evaluation of the measurement properties of the Epworth sleepiness scale: a systematic review," Sleep Medicine Reviews, vol. 18, no. 4, pp. 321-331, 2014.

[31] M. W. Johns, "Sensitivity and specificity of the multiple sleep latency test (MSLT), the maintenance of wakefulness test and the epworth sleepiness scale: failure of the MSLT as a gold standard," Journal of Sleep Research, vol. 9, no. 1, pp. 5-11, 2000.

[32] D. J. Buysse, M. L. Hall, P. J. Strollo et al., "Relationships between the Pittsburgh Sleep Quality Index (PSQI), Epworth Sleepiness Scale (ESS), and clinical/polysomnographic measures in a community sample," Journal of Clinical Sleep Medicine, vol. 4, no. 6, pp. 563-571, 2008.

[33] C. W. Newman, G. P. Jacobson, and J. B. Spitzer, "Development of the tinnitus handicap inventory," Archives of Otolaryngology-Head and Neck Surgery, vol. 122, no. 2, pp. 143148, 1996.

[34] A. McCombe, D. Baguley, R. Coles, L. McKenna, C. McKinney, and P. Windle-Taylor, "Guidelines for the grading of tinnitus severity: the results of a working group commissioned by the British Association of Otolaryngologists, Head and Neck Surgeons, 1999," Clinical Otolaryngology and Allied Sciences, vol. 26, no. 5, pp. 388-393, 2001.

[35] F. Zeman, M. Koller, R. Figueiredo et al., “Tinnitus handicap inventory for evaluating treatment effects: which changes are clinically relevant?" Otolaryngology: Head and Neck Surgery, vol. 145, no. 2, pp. 282-287, 2011.

[36] F. Zeman, M. Koller, M. Schecklmann et al., “Tinnitus assessment by means of standardized self-report questionnaires: psychometric properties of the Tinnitus Questionnaire (TQ), the Tinnitus Handicap Inventory (THI), and their short versions in an international and multi-lingual sample," Health and Quality of Life Outcomes, vol. 10, article 128, 2012. 
[37] J. A. Henry, T. L. Zaugg, and M. A. Schechter, "Clinical guide for audiologic tinnitus management I: assessment," American Journal of Audiology, vol. 14, no. 1, pp. 21-48, 2005.

[38] J. A. Henry, T. L. Zaugg, M. A. Schechter et al., How to Manage Your Tinnitus: A Step-by-Step Workbook, VA National Center for Rehabilitative Auditory Research, Portland, Ore, USA, 2008.

[39] J. A. Henry, T. L. Zaugg, P. J. Myers, and M. A. Schechter, “The role of audiologic evaluation in progressive audiologic tinnitus management," Trends in Amplification, vol. 12, no. 3, pp. 170-187, 2008.

[40] J. A. Henry, T. L. Zaugg, P. J. Myers et al., "Pilot study to develop telehealth tinnitus management for persons with and without traumatic brain injury," Journal of Rehabilitation Research and Development, vol. 49, no. 7, pp. 1025-1042, 2012.

[41] T. Zaugg, M. Schechter, S. Fausti et al., "Difficulties caused by patients' misconceptions that hearing problems are due to tinnitus," in Proceedings of the 7th International Tinnitus Seminar, Perth, Australia, 2002.

[42] G. Attanasio, F. Y. Russo, R. Roukos, E. Covelli, G. Cartocci, and M. Saponara, "Sleep architecture variation in chronic tinnitus patients," Ear and Hearing, vol. 34, no. 4, pp. 503-507, 2013.

[43] I. de Andrés, M. Garzón, and F. Reinoso-Suárez, "Functional anatomy of non-REM sleep," Frontiers in Neurology, vol. 2, article 70, 2011.

[44] S. Hébert, S. Fullum, and J. Carrier, "Polysomnographic and quantitative electroencephalographic correlates of subjective sleep complaints in chronic tinnitus," Journal of Sleep Research, vol. 20, no. 1, part 1, pp. 38-44, 2011.

[45] C. B. Saper, G. Cano, and T. E. Scammell, "Homeostatic, circadian, and emotional regulation of sleep," Journal of Comparative Neurology, vol. 493, no. 1, pp. 92-98, 2005.

[46] G. Cano, T. Mochizuki, and C. B. Saper, "Neural circuitry of stress-induced insomnia in rats," The Journal of Neuroscience, vol. 28, no. 40, pp. 10167-10184, 2008.

[47] L. W. Henselman, D. Henderson, J. Shadoan, M. Subramaniam, S. Saunders, and D. Ohlin, "Effects of noise exposure, race, and years of service on hearing in U.S. army soldiers," Ear and Hearing, vol. 16, no. 4, pp. 382-391, 1995.

[48] A. Axelsson and D. Prasher, "Tinnitus induced by occupational and leisure noise," Noise Health, vol. 2, no. 8, pp. 47-54, 2000.

[49] J. A. Henry, C. Loovis, M. Montero et al., "Randomized clinical trial: group counseling based on tinnitus retraining therapy," Journal of Rehabilitation Research \& Development, vol. 44, no. 1, pp. 21-32, 2007.

[50] C. Seydel, H. Haupt, H. Olze, A. J. Szczepek, and B. Mazurek, "Gender and chronic tinnitus: differences in tinnitus-related distress depend on age and duration of tinnitus," Ear and Hearing, vol. 34, no. 5, pp. 661-672, 2013.

[51] M. A. Fagelson, "The association between tinnitus and posttraumatic stress disorder," American Journal of Audiology, vol.16, no. 2, pp. 107-117, 2007.

[52] The Management of MDD Working Group, "VA/ DoD Clinical Practice Guideline for Management of Major Depressive Disorder (MDD)," 2009, http://www.healthquality.va.gov/ guidelines/MH/mdd/MDDFULL053013.pdf.

[53] F. H. Norris and L. B. Slone, "Understanding research on the epidemiology of trauma and PTSD," PTSD Research Quarterly, vol. 24, no. 2-3, p. 1, 2013.

[54] S. Salhofer, D. Lieba-Samal, E. Freydl, S. Bartl, G. Wiest, and C. Wöber, "Migraine and vertigo-a prospective diary study," Cephalalgia, vol. 30, no. 7, pp. 821-828, 2010.
[55] R. R. Rosa, M. H. Bonnet, and M. Kramer, "The relationship of sleep and anxiety in anxious subjects," Biological Psychology, vol. 16, no. 1-2, pp. 119-126, 1983.

[56] T. W. Uhde, B. M. Cortese, and A. Vedeniapin, "Anxiety and sleep problems: emerging concepts and theoretical treatment implications," Current Psychiatry Reports, vol. 11, no. 4, pp. 269276, 2009.

[57] J. B. S. Halford and S. D. Anderson, "Anxiety and depression in tinnitus sufferers," Journal of Psychosomatic Research, vol. 35, no. 4-5, pp. 383-390, 1991.

[58] D. J. Stein, N. Zungu-Dirwayi, G. J. van der Linden, and S. Seedat, "Pharmacotherapy for posttraumatic stress disorder," Cochrane Database of Systematic Reviews, no. 4, Article ID CD002795, 2000.

[59] B. Arroll, C. R. Elley, T. Fishman et al., "Antidepressants versus placebo for depression in primary care," Cochrane Database of Systematic Reviews, no. 3, Article ID CD007954, 2009.

[60] S. Wilson and S. Argyropoulos, "Antidepressants and sleep: a qualitative review of the literature," Drugs, vol. 65, no. 7, pp. 927947, 2005.

[61] P. Baldo, C. Doree, and P. Molin, "Antidepressants for patients with tinnitus," Cochrane Database of Systematic Reviews, vol. 9, p. CD003853, 2012. 


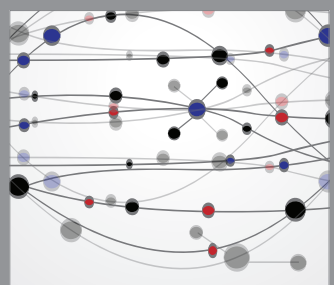

The Scientific World Journal
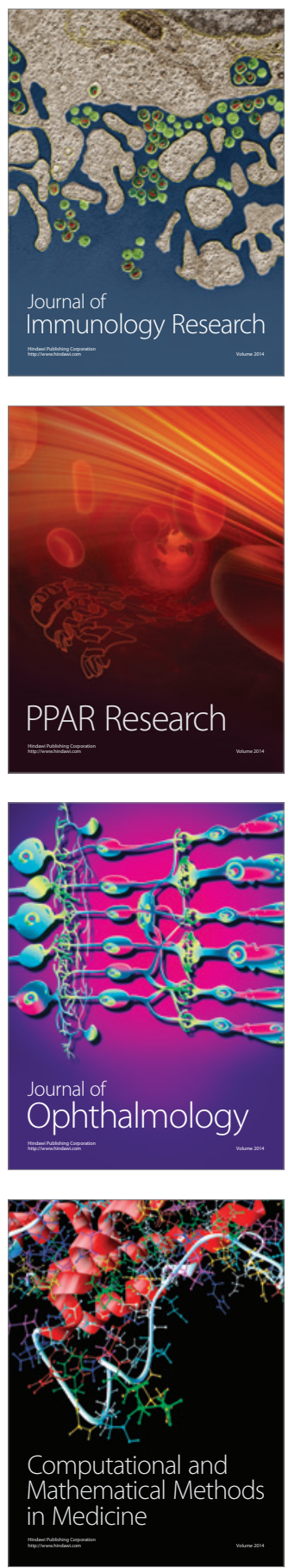

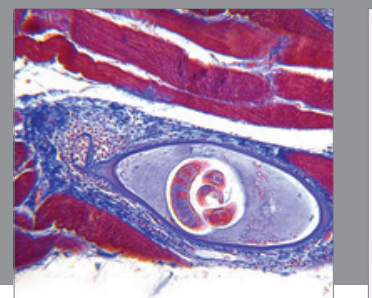

Gastroenterology

Research and Practice
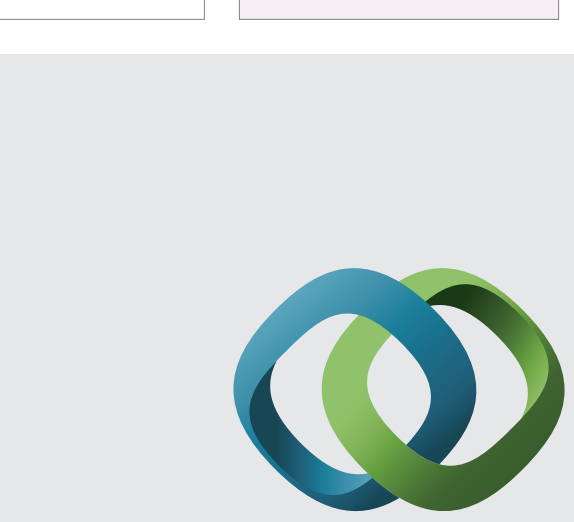

\section{Hindawi}

Submit your manuscripts at

http://www.hindawi.com
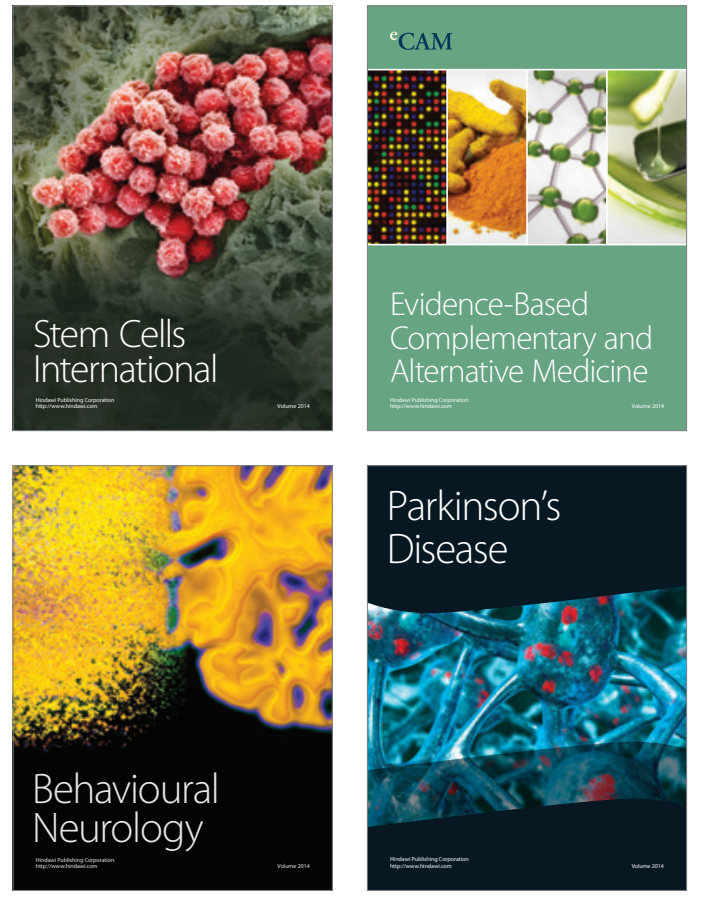
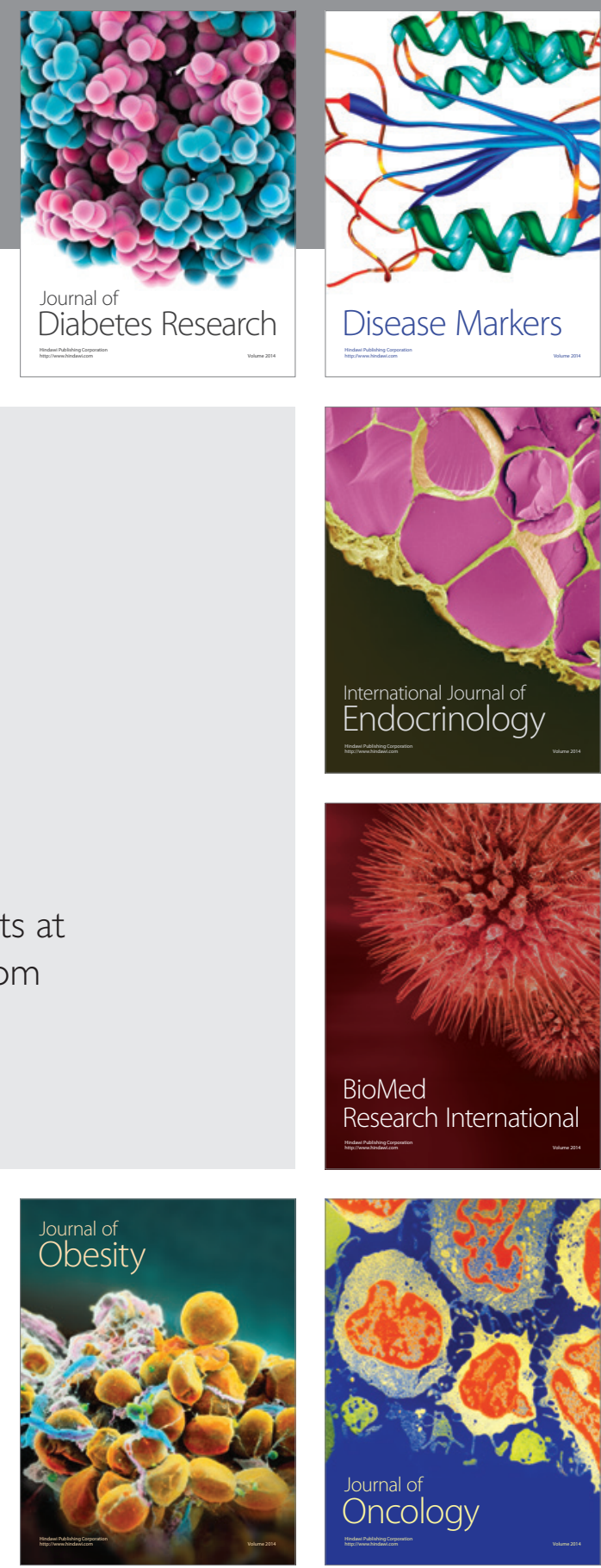

Disease Markers
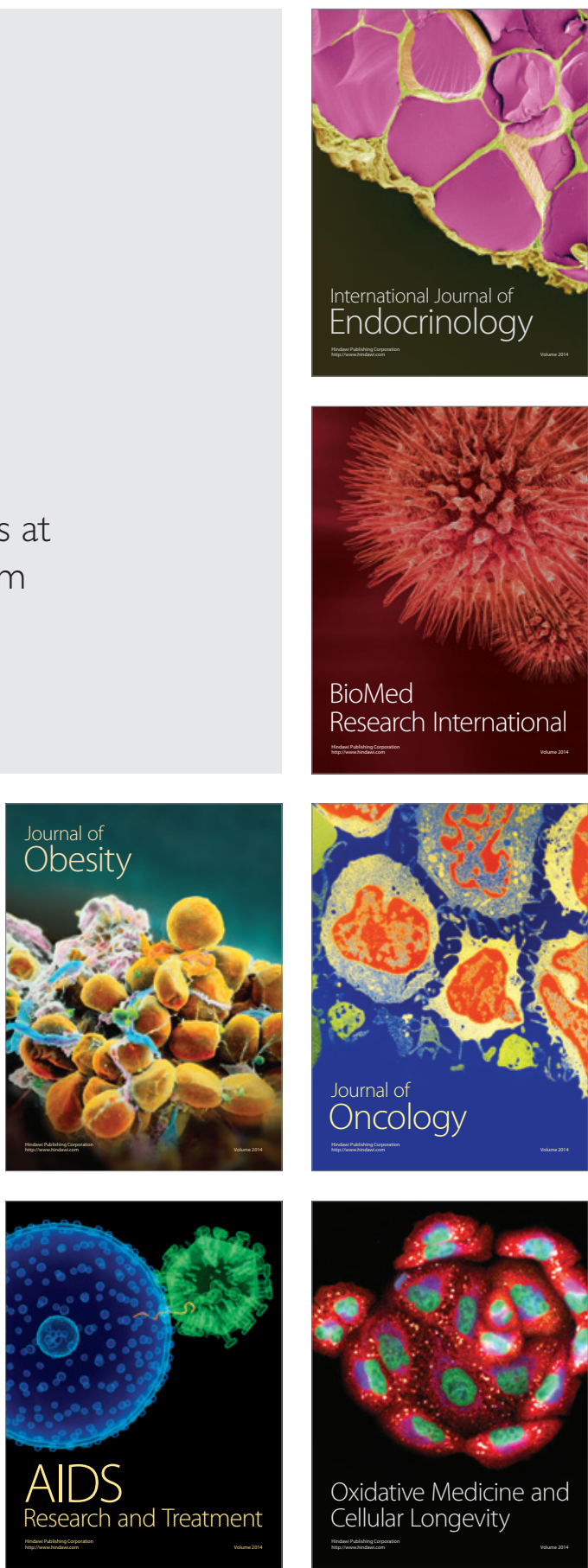